\title{
O TRABALHO EM EQUIPE NA INSTITUIÇÃO HOSPITALAR: UMA REVISÃO INTEGRATIVA
}

Silvia Helena Henriques Camelo ${ }^{1}$

\begin{abstract}
RESUMO: O hospital na atualidade constitui-se em organização de prestação de serviços de saúde onde congrega saberes, profissionais, tecnologia e infra-estrutura diversificados. O trabalho em equipe é uma estratégia que contempla a articulação das ações e dos saberes dos diversos profissionais inseridos neste ambiente, promovendo a integralidade do cuidado. Este artigo teve como objetivo contextualizar a modalidade de trabalho em equipe como dimensão cuidadora na instituição hospitalar. Trata-se de uma revisão integrativa realizada por meio de consulta às bases eletrônicas Lilacs e SciELO, o levantamento bibliográfico foi realizado entre os meses de agosto a novembro de 2010. Os resultados mostraram que os hospitais apresentam estrutura hierarquizada vertical e fragmentação das responsabilidades. O trabalho em equipe é utilizado para enfrentar o processo de especialização e ensejar a oportunidade de se construir uma visão global de cada situação mediante uma interlocução entre os profissionais envolvidos no seguimento terapêutico.
\end{abstract}

PALAVRAS-CHAVE: Hospitais; Assistência hospitalar; Equipe de assistência ao paciente.

\section{TEAMWORKING IN HOSPITAL INSTITUTIONS: AN INTEGRATIVE REVISION}

ABSTRACT: A present-day hospital is an organization for providing health services, where knowledge, health service professionals, technology, and a wide variety of infrastructure come together. Team-working is a strategy which aims at linking the actions and knowledge of the diverse health professionals inserted in this environment, promoting the integrality of care. This article aimed to contextualize the modality of teamwork with a caring dimension in a hospital institution. It is an integrative revision carried out by consulting the electronic databases Lilacs and SciELO; the bibliographic survey was effected between the months of August and November 2010. The results showed that hospitals present a structure with a vertical hierarchy and fragmentation of responsibilities. Teamwork is used to take on the process of specialization and occasion the opportunity to construct a global vision of each situation, through communication between the professionals involved in the therapeutic process. KEYWORDS: Hospitals; Hospital care; Patient care team.

\section{EL TRABAJO EN EQUIPO EN LA INSTITUCIÓN HOSPITALAR: UNA REVISIÓN INTEGRATIVA}

RESUMEN: En la actualidad, el hospital se constituye en una organización de prestación de servicios de salud, donde se congregan saberes, profesionales, tecnología e infraestructura diversificados. El trabajo en equipo es una estrategia que contempla la articulación de las acciones y de los saberes de los diversos profesionales de este ambiente, promoviendo la integralidad del cuidado. Este artículo tuvo como objetivo contextualizar la modalidad de trabajo en equipo como dimensión cuidadora y la institución hospitalar. Es una revisión integrativa realizada por medio de consulta a las bases electrónicas Lilacs y SciELO; la investigación bibliográfica fue realizada entre los meses de agosto y noviembre de 2010. Los resultados mostraron que los hospitales presentan estructura hierarquizada vertical y fragmentación de las responsabilidades. El trabajo en equipo es utilizado para afrontar el proceso de especialización y dar la oportunidad de construir una visión global de cada situación por medio del diálogo entre los profesionales involucrados en el sector terapéutico. PALABRAS-CLAVE: Hospitales; Asistencia hospitalar; Equipo de asistencia al paciente.

${ }^{1}$ Enfermeira. Doutora em Enfermagem. Professora do Departamento de Enfermagem Geral e Especializada da Escola de Enfermagem de Ribeirão Preto da Universidade de São Paulo. Membro do Grupo de Pesquisa Núcleo de Estudos sobre Saúde e Trabalho-NUESAT.

Universidade de São Paulo

Av. dos Bandeirantes, 3900 - 14040-902 - Ribeirão Preto-SP-Brasil

E-mail: shcamelo@eerp.usp.br

Cogitare Enferm. 2011 Out/Dez; 16(4):734-40 


\section{INTRODUÇÃO}

O trabalho que atende às necessidades de saúde é uma das formas mais antigas de trabalho social ${ }^{(1)}$. O trabalho em saúde é, hoje, majoritariamente coletivo institucional, e se desenvolve com características do trabalho Profissional, da divisão parcelar ou pormenorizada e da lógica taylorista de organização e gestão do trabalho ${ }^{(2)}$.

O ambiente hospitalar apresenta especificidades no mundo do trabalho em saúde. É formado por uma diversidade de profissionais ou trabalhadores treinados para realizar variadas atividades necessárias à manutenção da estrutura da instituição no que concerne ao conjunto de objetivos estabelecidos, sendo o trabalhador o sujeito da sua própria ação e da sua relação com o meio ${ }^{(3)}$.

O hospital, na atualidade constitui-se em uma organização social complexa e como tal, ocupa lugar crítico na prestação de serviços de saúde, sendo um lugar de construção de identidades profissionais, com grande reconhecimento social. O hospital congrega, assim, profissionais, saberes, tecnologias e infraestrutura diversificados ${ }^{(4)}$.

Sua configuração técnica e social é peculiar, caracterizada por uma divisão do trabalho extremamente precisa, com numerosos agentes que desenvolvem o trabalho em saúde, bem como promove a hierarquização de funções e tarefas. A forte concentração de poder decisório no topo da estrutura e o grande número de níveis hierárquicos dificultam, muitas vezes, o fluxo da comunicação e informação. O hospital é também um equipamento de saúde em processo de redefinição, pois tanto no âmbito público como no privado estão em debate o seu papel e seu lugar na produção do cuidado, em busca de qualidade, integralidade, eficiência e controle de custos ${ }^{(4)}$.

Atualmente, qualidade, produtividade e atenção à clientela são temas dominantes que visam o progresso e sobrevivência da organização hospitalar.

Na busca de uma melhor assistência à saúde, faz-se necessário o cumprimento das diretrizes e princípios do Sistema Único de Saúde (SUS) ${ }^{(5)}$. A integralidade da assistência - um desses princípios- é indispensável à qualidade da atenção à saúde e deve ser o eixo central na organização do trabalho nos hospitais. Dessa forma, o conceito ampliado de saúde, a responsabilização, a continuidade da atenção, a construção multiprofissional de projetos terapêuticos, e a busca pela autonomia dos usuários e de suas famílias na produção da saúde não são atributos exclusivos da Atenção Básica. Devem ser valores também presentes na organização do trabalho em saúde nos hospitais ${ }^{(4)}$.
Nesse sentido, torna-se fundamental articular as ações realizadas no interior da instituição hospitalar, de modo que ocorra a integração do trabalho dos diversos profissionais de uma mesma área e também entre equipes de trabalhadores de diferentes áreas.

O trabalho em equipe é uma estratégia de organização do trabalho que contempla, simultaneamente, a articulação das ações e dos saberes de diversas categorias profissionais em busca de consenso e que se traduz em qualidade na atenção integral às necessidades de saúde de sua clientela.

A organização do trabalho baseada na formação de equipes certamente tem sido a forma mais democrática, produtiva e humanizada de se realizar o trabalho em saú$\mathrm{de}^{(6)}$. As equipes devem ter autonomia para desenvolver novos projetos e métodos de trabalho, bem como sugerir políticas de pessoal para a organização do trabalho.

Com as mudanças recentes nas políticas de saúde, o mercado de trabalho vem sinalizando a necessidade de profissionais capazes de trabalhar em equipe, com conhecimentos, atitudes e habilidades que possibilitem 0 alcance de resultados esperados ${ }^{(7)}$.

Reconhecendo-se as mudanças pelas quais vêm passando as organizações hospitalares, são necessários estudos sobre a proposta do trabalho em equipe. Portanto, torna-se imperioso, para a gestão das pessoas, descrever como a estratégia do trabalho em equipe pode interferir na assistência integral prestada em âmbito hospitalar, por meio da qual os profissionais devem interagir e trabalhar com senso e liberdade de criação.

Assim, este artigo teve como objetivo contextualizar a modalidade do trabalho em equipe, como dimensão cuidadora na instituição hospitalar. Sua relevância consiste na possibilidade de conhecer a forma como está organizado o trabalho no ambiente hospitalar, provocando uma reflexão dos profissionais de saúde que atuam nos hospitais sobre a utilização da estratégia do trabalho em equipe na reestruturação das atividades de assistência, visando a qualidade na atenção à saúde.

\section{MÉTODO}

Para o alcance do objetivo, optamos pelo método da revisão integrativa, visto que é a mais ampla abordagem metodológica referente às revisões literárias, permitindo a inclusão de estudos experimentais e não experimentais para uma compreensão completa do fenômeno analisado, além de combinar dados da literatura teórica e empírica ${ }^{(8)}$. $\mathrm{Na}$ operacionalização dessa revisão utilizamos as seguintes etapas: seleção 
da questão temática, estabelecimento dos critérios para a seleção da amostra, representação das características da pesquisa original, análise dos dados, interpretação dos resultados e apresentação dos resultados da revisão.

A estratégia de busca ocorreu na Biblioteca Virtual em Saúde nas bases de dados caracterizadas como Literatura Latino-Americana e do Caribe em Ciências da Saúde-LILACS e Scientific Eletronic Library online-SciELO, e para o levantamento bibliográfico dos artigos, utilizamos os Descritores em Ciências da Saúde (DeCS): Hospitais, Assistência hospitalar e Equipe de assistência ao paciente. Foi utilizada também a palavra-chave "trabalho em equipe". Os descritores e a palavra-chave foram combinados entre si, de acordo com a base de dados.

Os critérios de inclusão utilizados para a seleção da amostra foram: artigos publicados em português, inglês e espanhol, nos últimos seis anos, disponíveis na íntegra nas bases de dados selecionadas, e que abordassem a temática do trabalho em equipe em âmbito hospitalar. $\mathrm{O}$ levantamento foi realizado entre os meses de agosto a novembro de 2010 e os resultados foram analisados por meio da abordagem qualitativa dos dados.

\section{RESULTADOS}

Os artigos encontrados nas bases de dados, conforme descritores estabelecidos, estão em língua portuguesa, totalizando oito artigos; foram publicados no período de 2004 a 2009 em revistas nacionais, algumas de circulação internacional, com predominância de periódicos vinculados às universidades públicas. $\mathrm{Na}$ produção dos artigos houve a participação de mestres, doutores em enfermagem, docentes de universidades públicas e privadas, enfermeiros assistenciais e estudantes de graduação em Enfermagem. Os descritores mais utilizados pelos autores foram "equipe de enfermagem", "equipe de assistência ao paciente" e "assistência integral à saúde".

Ao analisarmos os delineamentos de pesquisa mais frequentes na amostra estudada, identificamos que todos utilizaram a abordagem metodológica qualitativa para descrição dos seus resultados. Esse tipo de abordagem em pesquisa é um meio de gerar conhecimentos sobre fenômenos subjetivos que constituem foco de interesse da profissão.

$\mathrm{Na}$ análise destes artigos foram consideradas as informações referentes a consensos da Organização Mundial de Saúde, livros e outros estudos que abordam a temática do trabalho em equipe e a organização hospitalar.
Assim, a análise temática do material coletado sobre o tema investigado revelou três categorias: $a$ organização do trabalho na instituição hospitalar; a estratégia do trabalho em equipe e dimensão cuidadora do trabalho em equipe no hospital.

Na primeira categoria, destaca-se o papel dos hospitais enquanto instituição social complexa inserida no atual modelo de atenção à saúde; a seguir, apresentamse conceitos e tipologias do trabalho em equipe e a possibilidade de resultados satisfatórios desta proposta no contexto hospitalar. Finalmente, a última categoria aborda o trabalho em equipe como estratégia para a integralidade do cuidado em âmbito hospitalar.

\section{DISCUSSÃO}

\section{Organização do trabalho na instituição hospitalar}

O caminho que o hospital percorre em sua história como instituição aponta para a sua transformação de depositário de doentes e excluídos em locus de extrema concentração de tecnologia e saber ${ }^{(9)}$.

Sendo o hospital, desde sua gênese, espaço de formação médica, ocorre também em seu ambiente o processo de acumulação de conhecimentos pela experiência, trocas e relações de solidariedade, o que constitui uma vertente positiva, no sentido de desfazer, em termos relativos, a fragmentação dos conhecimentos sobre as pessoas e suas doenças ou sobre as pessoas e seus relacionamentos em um hospital específico. Mais do que os espaços de formação formalmente constituídos, e conhecimento empírico acumulado, na prática, os hospitais ampliam a possibilidade de trocas e diminuem a distância entre as pessoas ${ }^{(9)}$.

Segundo a Organização Mundial de Saúde (OMS), o novo papel dos hospitais nos sistemas integrados de serviços de saúde exige deles um conjunto de características como: ser um lugar para manejo de eventos mórbidos agudos; deve ser utilizado exclusivamente em casos em que haja possibilidades terapêuticas; deve apresentar uma densidade tecnológica compatível com suas funções, o que significa ter unidades de tratamento intensivo e semi-intensivo, unidades de internação, centro cirúrgico, unidade de emergência, unidade de assistência farmacêutica, unidade de atenção ambulatorial, unidade de hospital-dia, unidade de atenção domiciliar terapêutica, etc; deve ter uma escala adequada para operar com eficiência e qualidade. Além disso, o hospital deve ter um projeto arquitetônico compatível com as suas funções e amigável aos seus usuários ${ }^{(10)}$. 
A estrutura organizacional dos hospitais segue, nos dias de hoje, as diretrizes estabelecidas por organogramas clássicos, com as estruturas hierarquizadas verticais, a fragmentação das responsabilidades e a formalização das relações, pautando-se nas lógicas de autoridade legal, herdadas da concepção burocrática. Estes modelos clássicos de gerenciamento influenciaram a administração de recursos humanos em saúde, nas últimas décadas, e têm levado as organizações a resultados insatisfatórios e ineficientes, pois não respondem às necessidades atuais ${ }^{(11)}$. A descentralização promovida pela implantação do SUS, no Brasil, as mudanças no modelo assistencial, bem como outros fatores como o uso intensivo de tecnologia de ponta, flexibilização das relações de trabalho e outros, vêm exigem um gerenciamento voltado para novos desafios ${ }^{(12)}$.

A perspectiva de construção de organogramas mais horizontais, de um modelo de gestão mais participativo nestas instituições, implica em reunir os profissionais naquilo que os mesmos têm em comum: o objeto de trabalho ${ }^{(11)}$. A atenção ao usuário, por meio da estratégia do trabalho em equipe, é uma proposta capaz de reunir grupos profissionais com interesses e lógicas de organização de trabalho diferenciadas, com vistas à construção de uma proposta assistencial o mais consensual possível.

O hospital é um ambiente onde se desenvolve um grande volume de atividades, que devem operar em harmonia; é o palco onde trabalham profissionais de várias especialidades e diferentes matizes. $\mathrm{O}$ comum entre eles é a atuação em perfeita consonância para a conquista dos resultados preconizados. Os serviços que esta instituição desenvolve devem interagir entre si com muita perfeição. Caso contrário, o objetivo final - a assistência de qualidade aos doentes - será prejudicada ${ }^{(13)}$.

\section{A estratégia do trabalho em equipe}

A proposta do trabalho em equipe é uma estratégia utilizada para enfrentar o intenso processo de especialização na área da saúde e na instituição hospitalar, pois esse processo tende a aprofundar verticalmente o conhecimento e a intervenção em aspectos individualizados das necessidades de saúde, sem contemplar, simultaneamente, a articulação das ações e dos saberes.

O trabalho em equipe consiste em uma modalidade de trabalho coletivo que se configura na relação recíproca entre intervenções técnicas e a interação dos agentes. No processo de trabalho em saúde, os profissionais constroem consensos que configuram um projeto assistencial comum, em torno do qual se dá a integração da equipe de trabalho ${ }^{(14)}$. Este tipo de trabalho enseja a oportunidade de se construir uma visão global de cada situação, mediante uma interlocução entre os diferentes profissionais envolvidos no seguimento terapêutico, contribuindo para alcançar um melhor prognóstico para os pacientes hospitalizados. Profissionais com formação e tempo de experiência diferentes podem contribuir de forma diversa para o desenvolvimento do trabalho em equipe ${ }^{(15)}$.

De acordo com a literatura, observa-se a distinção entres duas noções que recobrem a ideia de equipe: a equipe como agrupamento de agentes e a equipe como integração de trabalhos. A primeira é caracterizada pela fragmentação e a segunda pela articulação consoante à proposta da integralidade das ações de saúde ${ }^{(14)}$.

No contexto hospitalar, entende-se que um cuidado oferecido pela equipe multiprofissional, em que falta o sentido de integralidade poderá gerar resultados insatisfatórios para o indivíduo, família e sociedade ${ }^{(16)}$. Corroborando este fato, em estudo selecionado, profissionais de saúde do âmbito hospitalar destacaram a atuação em equipe como um dos elementos capazes de tonar eficaz e integral a sua assistência ${ }^{(17)}$.

Pensar no trabalho em equipe, no contexto hospitalar, significa pensar no cuidado centrado no paciente e suas necessidades, e não nas necessidades das categorias profissionais, pois a diversidade de situação, muitas vezes, exige respostas imediatas, sob pena de colocar em risco a vida do paciente. Assim, a integração de diferentes categorias profissionais e ramos do conhecimento em torno de um objetivo comum tornase fundamental para que a assistência ao cliente seja oportuna e livre de riscos ${ }^{(18)}$

Entretanto, alguns fatores podem interferir na integração dos profissionais e, dentre eles, destaca-se a atuação transitória de um grande número de estudantes de graduação e pós-graduação que desenvolvem atividades profissionalizantes, especialmente em hospitais de ensino ${ }^{(17)}$. A alta rotatividade dos profissionais é um dos principais desafios para a efetivação do trabalho em equipe, pois as idas e vindas dos profissionais dificultam a integração e construção de vínculos entre eles, assim como a consecução de objetivos comuns capazes de solucionar os problemas de saúde, de forma integral ${ }^{(19)}$.

Observa-se que ocorre maior integração na equipe quando há menor desigualdade entre os diferentes trabalhos e os respectivos agentes. Na medida em que o trabalho em equipe é construído, efetivamente, na relação intrínseca entre agentes do trabalho, quanto 
mais próximo for o estatuto de sujeito ético-social desses agentes, maiores as possibilidades de eles interagirem em situações livres de coação e de submissão, na busca de consensos acerca da finalidade e do modo de executar o trabalho ${ }^{(14)}$.

Somente um trabalho de efetiva integração e o estabelecimento de interrelações entre as diversas disciplinas proporcionarão a criação de condições propícias para enfrentar a complexidade do cuidar pensado com integralidade ${ }^{(20)}$.

\section{A dimensão cuidadora do trabalho em equipe no hospital}

A recuperação da dimensão cuidadora e a busca da integralidade na atenção à saúde são desafios colocados para a organização do cuidado no interior dos hospitais ${ }^{(21)}$.

Dentro do hospital, a atenção integral depende da conjugação do trabalho de vários profissionais, ou seja, o cuidado recebido pelo paciente é produto de um grande número de pequenos cuidados parciais que vão se complementando, a partir da interação entre os vários cuidadores que operam no hospital ${ }^{(4)}$.

Dessa forma, uma complexa trama de atos, procedimentos, fluxos, rotinas e saberes, num processo de complementação e disputa, compõem o cuidado em saúde. Ou seja, a forma como se articulam as práticas dos trabalhadores do hospital confere maior ou menor integralidade à atenção produzida ${ }^{(21)}$. Portanto, um importante desafio no processo gerencial do hospital é conseguir coordenar adequadamente este conjunto diversificado e especializado, de modo que eles resultem em um cuidado coordenado eficaz e de qualidade ${ }^{(22)}$.

A institucionalização das práticas de saúde, a perda de controle sobre os meios de trabalho, a crescente especialização e a maciça incorporação de tecnologias duras (equipamentos e medicamentos) contribuíram para que houvesse um predomínio das tecnologias duras e leve-duras (os saberes científicos da clínica e da epidemiologia) no trabalho em saúde ${ }^{(4)}$. Desse modo, o trabalho médico e o trabalho em saúde, em geral, orientaram-se mais para os procedimentos e menos para as necessidades das pessoas, havendo empobrecimento de sua dimensão cuidadora ${ }^{(23)}$.

Há um crescente debate mundial acerca das melhores maneiras de organizar a atenção à saúde num hospital. A busca pela humanização da atenção à saúde, qualidade e menores custos compõem as vertentes que operam em favor de uma reorganização da atenção à saúde, todas elas prevendo novos lugares e papeis para os hospitais da rede de serviços de saúde ${ }^{(4)}$.
Em nossa compreensão, o trabalho em equipe é o que mais se aproxima da dimensão da complexidade que caracteriza os cuidados em saúde, como contraresposta à crescente compartimentalização do saber, numa tentativa de superação do enfoque biomédico, curativista e fragmentado que tem sido adotado nas organizações hospitalares.

O trabalho em equipe só é possível quando os trabalhadores constroem uma interação entre si, trocando conhecimentos e articulando um campo de produção do cuidado, já que este é comum à maioria dos trabalhadores de um hospital. Esse campo de cuidado, além da interação, possibilita, a cada um, usar todo o seu potencial criativo na relação com o usuário/paciente, para juntos produzirem o cuidado ${ }^{(24)}$. Na produção de um ato de saúde coexistem vários núcleos, descritos como cuidadores, profissional específico e específico por problemas, sendo que todos os trabalhadores de saúde fazem clínica cuidadora e operam práticas sanitárias, independente do recorte profissional, o que proporciona pensar outras possibilidades no modo de agir em saúde ${ }^{(23)}$.

Apesar das discussões acerca do trabalho em equipe serem tão comuns nos ambientes de trabalho, na área da saúde, muitos profissionais ainda têm dificuldade de compreendê-lo e executá-lo, já que realizam ações parceladas e, muitas vezes, desarticuladas, mesmo sabendo que só por meio da articulação das ações e interação entre os membros da equipe é que se vai caminhar para um trabalho que consiga atender as necessidades multidimensionais dos indivíduos de quem se cuida ${ }^{(25)}$.

Os novos modelos de organização da assistência à saúde buscam estimular a construção de equipes, ao permitir a recomposição dos meios de trabalho, a reestruturação das atividades dos agentes e a redefinição das relações sociais e técnicas sob as quais se realizam os trabalhos ${ }^{(26)}$.

\section{CONSIDERAÇÕES FINAIS}

Esta pesquisa pretendeu ampliar a discussão acerca da estratégia do trabalho em equipe como dimensão cuidadora na instituição hospitalar.

O trabalho hospitalar exige novas competências dos profissionais, que se deparam com mudanças tecnológicas e exigências de sua clientela, provocando, muitas vezes, transformações no seu processo de trabalho. $\mathrm{O}$ hospital é um local de atuação de profissionais com diferentes níveis de formações, onde a estratégia do trabalho em equipe torna-se uma proposta que enfatiza a cooperação e incorpora a participação dos profissio- 
nais, fortalecendo o trabalho multiprofissional. Esta proposta é pensada, portanto, como um campo fértil para aquisição de habilidades e competências para o atendimento integral à saúde.

Os estudos analisados consideram a equipe um reservatório social de recursos, criando e recriando possibilidades, na medida em que a equipe possa se recriar a cada encontro. Nesse sentido, o trabalho em equipe é uma forma de articular as ações de saúde no hospital, integrar os agentes, superar o isolamento dos saberes e democratizar as relações de trabalho. É uma ferramenta ímpar, seja para o alcance dos bons resultados terapêuticos, seja para o avanço do tratamento em âmbito hospitalar.

Os profissionais que integram uma equipe interdisciplinar na organização hospitalar são participantes privilegiados para a compreensão da complexidade envolvida no processo saúde-doença. A modalidade do trabalho em equipe deve estabelecer relações entre os trabalhadores que favoreçam a realização de um trabalho integrado, garantindo qualidade no atendimento prestado.

Enfatizamos que, apesar das limitações, todo estudo desta natureza provoca reflexões nos trabalhadores de saúde que atuam em âmbito hospitalar - bem como dos administradores - sobre as potencialidades da estratégia do trabalho em equipe. Esta pressupõe transformações na organização do cuidado e na formação dos profissionais para atenção integral do paciente.

\section{REFERÊNCIAS}

1. Milhomem MIL, Oliveira MAGC. O trabalho em equipe nos centros de atenção psicossocial. Cogitare Enferm. 2007;12(1):101-8.

2. Ribeiro EM, Pires D, Blank VLG. A teorização sobre processo de trabalho em saúde como instrumental para análise do trabalho no programa saúde da família. Cad Saúde Pública. [Internet] 2004;20(2) [acesso em 23 set 2008]. Disponível: http://www.scielo.br/pdf/csp/v20n2/11.pdf

3. Alam MM, Cezar-Vaz MR, Almeida T. Educação ambiental e o conhecimento do trabalhador em saúde sobre situações de risco. Ciênc Saúde Coletiva. 2005;10 Suppl 0: 39-47.

4. Fueerwerker LC, Cecílio LCO. O hospital e a formação em saúde: desafios atuais. Rev C S Col. 2007;12(4):965-71.

5. Pinho IC, Siqueira JCBA, Pinho LMO. As percepções do enfermeiro acerca da integralidade da assistência. REE. 2006;8(1):42-51.
6. Spagnol CA, Ferraz CA. Tendências e perspectivas da administração em enfermagem: um estudo na Santa Casa de Belo Horizonte-MG. Rev Latino-Am Enfermagem. 2002;10(1):15-20.

7. Alves M, Mello RA. Trabalho em equipe entre profissionais da enfermagem em um centro de terapia intensiva. Ciênc Cuid Saúde. 2006;5(3):299-308.

8. Whittemore R, Knafl K. The integrative review: update methodology. J Adv Nurs. 2005;52(5):546-53.

9. Machado JMH, Correa MV. Conceito de vida no trabalho na análise das relações entre processo de trabalho e saúde no hospital. Inf Epidemiol SUS. 2002;11(3):159-66.

10. World Health Organization 2000. The world health reports 2000: health systems, improving performance. WHO. Genebra.

11. Matos E, Pires D. Teorias administrativas e organização do trabalho: de Taylor aos dias atuais, influências no setor saúde e na enfermagem. Texto Contexto Enferm. [Internet] 2006;15(3) [acesso em 17 set 2008]. Disponível: http://www.scielo.br/pdf/tce/v15n3/ v15n3a17.pdf

12. Castro JL. Nova agenda para a administração de recursos humanos de saúde. Texto de Apoio/ Unidade 3. [Internet] [acesso em 06 jan 2006]. Disponível: http://www.opas.org.br/rh/publicacoes/textos_apoio/ pub04U3T3.pdf

13. Cherubin NA. A arte de ser administrador hospitalar líder. São Paulo: Centro Universitário São Camilo; 2003.

14. Peduzzi M. Equipe multiprofissional de saúde: conceito e tipologia. Rev Saúde Públ. [Internet] 2001:35(1) [acesso 22 nov 2010]. Disponível: http://www.scielosp. org/pdf/rsp/v35n1/4144.pdf

15. Queiroz E, Araújo TCCF. Trabalho de equipe em reabilitação: um estudo sobre a percepção individual e grupal dos profissionais de saúde. Paidéia. 2009;19(43):177-87.

16. Duarte ED, Sena RR, Xavier CC. Processo de trabalho na unidade de terapia intensiva neonatal: construção de uma atenção orientada pela integralidade. Rev Esc Enferm USP. [Internet] 2009:43(3) [acesso em 04 mar 2011]. Disponível: http://www.scielo.br/pdf/reeusp/ v43n3/a21v43n3.pdf 
17. Santos DL, Santos JLG, Prochnow AG, Pedroso MLR, Lima MADS. A integralidade nas ações da equipe de saúde de uma unidade de internação pediátrica. Interface: Comunic Saúde Educ. [Internet] 2009:13(31) [acesso em 04 mar 2004]. Disponível: http://www.lume. ufrgs.br/bitstream/handle/10183/27380/000730054. pdf?sequence $=1$

18. Alves M, Ramos FRS, Penna CMM. O trabalho interdisciplinar: aproximações possíveis na visão de enfermeiras de uma unidade de emergência. Texto Contexto Enferm. [Internet] 2005:14(3) [acesso em 04 mar 2011]. Disponível: http://www.scielo.br/pdf/tce/ v14n3/v14n3a02.pdf

19. Colomé ICS, Lima MADS. Desafios do trabalho em equipe para enfermeiras que atuam no Programa Saúde da Família (PSF). Rev Gaúch Enferm. 2006;27(4):54856.

20. Silva LM, Santos MA. Construindo pontes: relato de experiência de uma equipe multidisciplinar em transtornos alimentares. Medicina. 2006;39(3):415-24.

21. Cecílio LCO, Merhy EE. A integralidade do cuidado como eixo da gestão hospitalar. In: Pinheiro R, Matos R. Construção da integralidade: cotidiano, saberes e práticas em saúde. Rio de Janeiro: IMS/Abrasco; 2003.

22. Merhy EE, Cecílio LCO. O singular processo de coordenação dos hospitais. Saúde em Debate. 2003;22(64):110-22.

23. Merhy EE. Saúde: a cartografia do trabalho vivo em ato. São Paulo: Hucitec; 2002.

24. Franco TB, Merhy EE. O uso de ferramentas analisadoras para apoio ao planejamento dos serviços de saúde: o caso do serviço social do hospital das clínicas da UNICAMP. In: Merhy EE, Magalhães Júnior HM, Rimoli J, Franco TB, Bueno WS. O trabalho em saúde: olhando e experienciando o SUS no cotidiano. São Paulo: Hucitec; 2003.

25. Gaíva MAM, Scochi CGS. Processo de trabalho em saúde em enfermagem neonatal e UTI. Rev Latino-Am Enfermagem. [Internet] 2004:12(3) [acesso em $04 \mathrm{mar}$ 2011]. Disponível: http://www.scielo.br/pdf/rlae/v12n3/ v12n3a04.pdf

26. Araújo MBS, Rocha PM. Trabalho em equipe: um desafio para a consolidação da estratégia de saúde da família. Cad Saúde Pública. 2007;12(2):455-64. 